\title{
Randomised controlled trial using daily electrical muscle stimulation (EMS) in critically ill patients to prevent intensive care unit (icu) acquired weakness (ICUAW)
}

\author{
M Goll, , T Wollersheim, K Haas, R Moergeli, J Malleike, F Nehls, K Reiher, N Carbon, G Sonomoya, \\ $S$ Weber-Carstens
}

From ESICM LIVES 2015

Berlin, Germany. 3-7 October 2015

\section{Introduction}

Critically ill patients frequently suffer from intensive care unit (ICU) acquired weakness (ICUAW). Evoke muscle contraction by electrical muscle stimulation (EMS) may help to prevent their overall strength diminishing by conserving muscle strength and mass.

\section{Objectives}

The aim of the study was to investigate the effects of daily EMS-therapy on muscular strength in ICU patients, compared to a protocol based standard physiotherapy.

\section{Methods}

The interventional randomized controlled trial included critically ill patients at high risk for ICUAW selected by SOFA score $\geq 9$ and onset of critical illness in less than 72 hours. Randomization was administered to EMS group (protocol base physiotherapy and additional EMS) or a physiotherapy group (protocol base physiotherapy). EMStherapy was applied for 20 minutes 7 days/week to 8 bilateral muscle groups by an experienced physiotherapist. Medical-Research-Council (MRC) score for muscle strength and grip strength measured by hand-dynamometer were recorded on the first day patients were adequately awake and on the day of their ICU discharge. Non-parametric tests were performed. Ethic vote (Charité EA 2/041/10).

\section{Results}

50 patients, 33 males and 17 females with a median age of $52(10 / 90)$ years and a SOFA score of $13(10 / 15)$ at

Charité - Universitätsmedizin Berlin, Berlin, Germany admission where included in the study. Randomization resulted in 21 to 29 subjects for EMS and controls respectively. Time-points of muscle strength measurements were "first day being awake" at median day $11(7 / 19)$ and discharge form ICU, median day 26 (17/33). Patient characteristics and time-points of recorded parameters were without significant differences between the intervention (EMS) and control group (data not shown). Overall subjects fulfilled criteria for ICUAW on their first day of being awake and significantly improved their MRC score and grip strength until their ICU discharge. Surprisingly we could not show direct group differences between EMSand control group for MRC score and grip strength. By focusing separately on the course of strength for EMS- and control group we found an increase in MRC score for both groups between the first and second measurement.

Grip strength in nonEMS treated patients rose significantly whilst patients receiving EMS couldn't show significant improvements between measurements.

\section{Conclusions}

Our patients managed to increase their grip- and general strength levels significantly from their first day of being adequately awake until the day of their ICU discharge. Further subgroup analysis however indicates that EMS therapy may not be more beneficial in increasing grip or MRC-measured strength compared to the standard physiotherapy protocol within our study. The missing grip strength improvement of the EMS group between the first day of being awake and ICU discharge may be attributed to greater muscle strength at time of awakening without significant power to show group differences. 

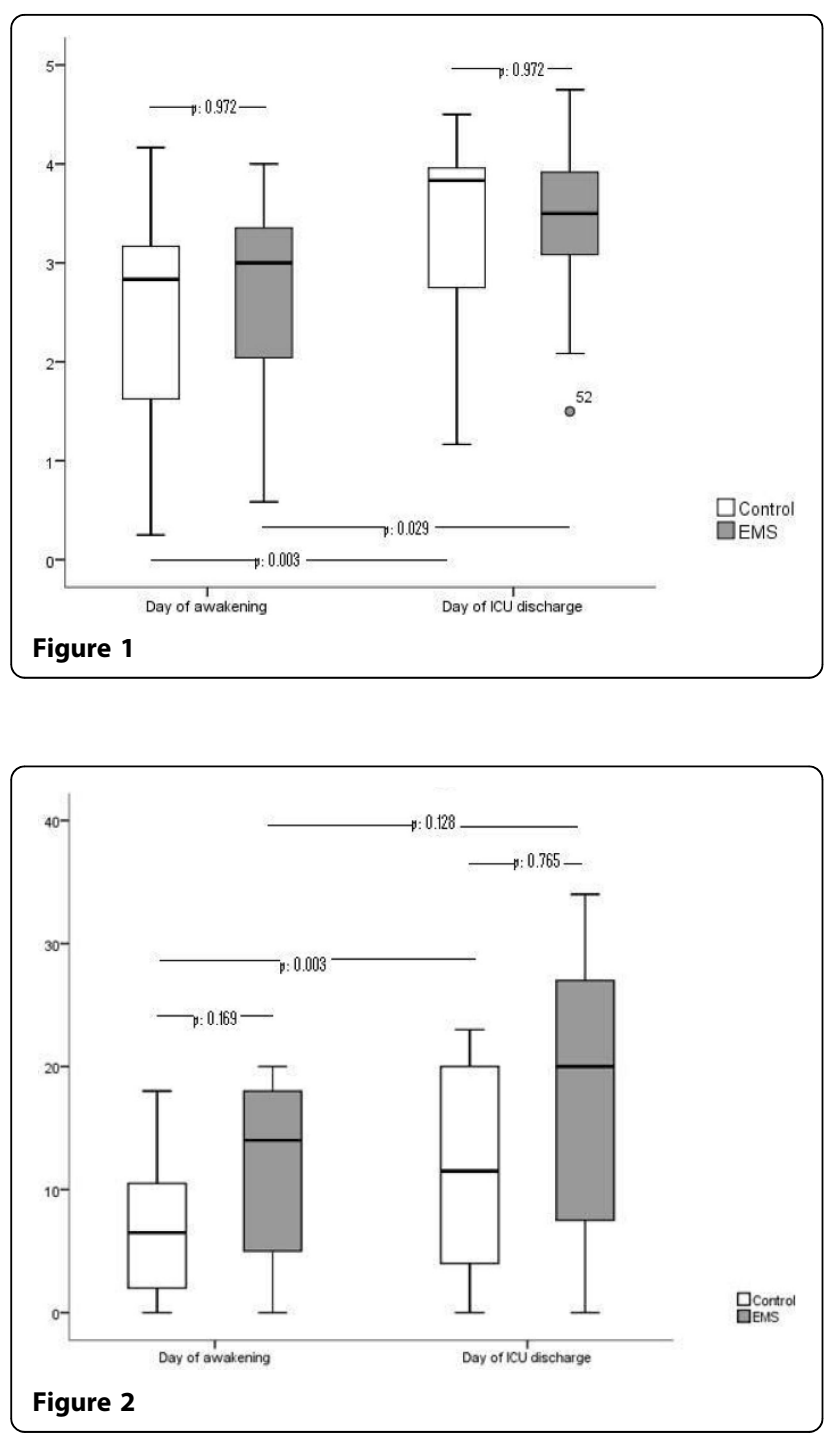

\section{Grant Acknowledgment}

DFG KFO 192/2 TP3.

Published: 1 October 2015

doi:10.1186/2197-425X-3-S1-A809

Cite this article as: Goll et al:: Randomised controlled trial using daily electrical muscle stimulation (EMS) in critically ill patients to prevent

intensive care unit (icu) acquired weakness (ICUAW). Intensive Care Medicine Experimental 2015 3(Suppl 1):A809.

\section{Submit your manuscript to a SpringerOpen ${ }^{\mathcal{O}}$ journal and benefit from:}

- Convenient online submission

- Rigorous peer review

- Immediate publication on acceptance

- Open access: articles freely available online

- High visibility within the field

- Retaining the copyright to your article

Submit your next manuscript at $>$ springeropen.com 